\title{
Note sur la valeur azotée de fourrages pauvres traités par l'urée ou l'ammoniac
}

\author{
H. BENAHMED, J.P. DULPHY \\ avec la collaboration technique de Madeleine DUDILIEU, Marie JAILLER, \\ Jacqueline JAMOT, H. BOUSQUET et L. L'HOTELIER \\ I.N.R.A., Laboratoire des Aliments \\ F 63122 Ceyrat
}

\begin{abstract}
Résumé
Un foin tardif de prairie naturelle et une paille de blé ont été traités, sous bâche, soit avec de l'ammoniac (3 p. 100), soit avec de l'urée (5 p. 100). Les fourrages non traités et traités ont été ensuite distribués à des moutons normaux ou fistulisés du rumen. Avec la paille de blé non traitée, les moutons ont reçu en outre $100 \mathrm{~g}$ de tourteau de soja par jour.

Le traitement à l'ammoniac a permis d'augmenter nettement la teneur en MAT des fourrages $(+85$ et $+60 \mathrm{~g} / \mathrm{kg}$ de MS), les quantités ingérées $(+26$ et $+9 \mathrm{p} .100)$ et la digestibilité de la matière organique $(+7,9$ et $+7,7$ points $)$.

Par contre le traitement à l'uréc n'a pas été efficace. La teneur en MAT des fourrages a augmenté $(+111$ et $+48 \mathrm{~g} / \mathrm{kg}$ de MS) mais les quantités ingérées ont diminué de 17 p. 100 pour la paille et la digestibilité de la matière organique n'a pas été modifiée. Cela est dû principalement au fait que l'urée ne s'est pas suffisamment transformée en ammoniac (19 p. 100 seulement en moyenne pour les 2 fourrages).

Avec le foin, les traitements - surtout celui à l'ammoniac - ont abaissé l'activité cellulolytique dans le rumen. Curieusement on a observé l'inverse avec la paille.

Le traitement à l'ammoniac a eu pour conséquence une baisse de la rétention azotée par les animaux.

Il semble donc bien que la valeur azotée des fourrages traités à l'ammoniac, malgré un apport important d'azote, soit relativement faible, mais la dépression de l'activité cellulolytique entrânée par le traitement n'est peut-être pas systématique.

Le traitement à l'urée, dont l'étude se développe par ailleurs et qui donne de bons résultats, devra être adapté à nos fourrages et à nos conditions de traitement.
\end{abstract}

Mots clés : Foin, paille, traitement à l'ammoniac, valeur alimentaire, rétention azotée.

\section{Introduction}

Les fourrages pauvres ont fait l'objet de nombreux travaux en vue d'améliorer leur ingestibilité et leur valeur nutritive par des traitements divers. Les traitements chimiques sont maintenant couramment utilisés et donnent de bons résultats. Après le traitement à la soude (JAckson, 1977) celui à l'ammoniac (Sundstøl, Coxworth \& Mowat, 1978) s'est largement développé dans les Pays Scandinaves et en France. 
Le traitement par l'ammoniac est surtout appliqué aux pailles, mais il peut l'être également pour des foins de faible valeur (DulPHy et al., 1984). Pour les pailles il est intéressant car, en plus de l'augmentation de l'ingestibilité et de la digestibilité de la matière organique, il est susceptible d'apporter de l'azote non protéique et d'améliorer la valeur azotée.

Dans les études que nous avons entreprises sur le traitement à l'ammoniac, deux problèmes concernant la valeur nutritive des fourrages après traitement nous sont apparus (Dulphy et al., 1984 ; Dulphy, Komar, Zwaenepoel, 1984) :

- celui de la valeur azotée réelle des fourrages traités;

- celui de l'activité cellulolytique qu'ils induisent dans le rumen.

C'est pourquoi, dans un nouvel essai, avec un foin tardif et une paille traités ou non à l'ammoniac nous avons mesuré l'azote retenu par les moutons recevant les fourrages. Nous avons, par ailleurs, expérimenté un autre traitement, celui à l'urée. En effet, l'urée introduit dans le fourrage en enceinte hermétique peut générer de l'ammoniac (Hadjipanayiotou, 1982 ; Cloete, DE Villiers \& Kritzinger, 1983) et améliorer la digestibilité de ce fourrage.

\section{Matériel et méthodes}

Nous avons utilisé deux fourrages pauvres : un foin tardif de prairie naturelle et une paille de blé. Ces deux fourrages, conditionnés en balles de moyenne densité $(12-15 \mathrm{~kg} /$ balle), ont été traités, pour partie, soit avec $3 \mathrm{p} .100 \mathrm{~d}$ 'ammoniac, soit avec 5 p. 100 d'urée, urée en solution concentrée à $500 \mathrm{~g} /$ litre. Les traitements ont été effectués en tas de $300 \mathrm{~kg}$ recouverts hermétiquement d'une bâche plastique, selon la méthode décrite par Sundstøl, Coxworth \& Mowat (1978) pour l'ammoniac ou par addition (arrosage) de la solution d'urée dans les tas au fur et à mesure de leur confection. Les traitements ont eu lieu le 9 septembre 1983 et les tas ont été débâchés le 19 décembre 1983, puis laissés découverts 2 semaines avant la distribution des fourrages aux animaux.

Les 6 fourrages ainsi préparés ont été distribués à 16 béliers castrés de race Texel, âgés de 2 ans environ et pesant $55 \mathrm{~kg}$ en moyenne. Quatre d'entre eux étaient porteurs d'une fistule du rumen.

Six moutons non fistulisés, par groupe de 2, ont reçu les 3 foins successivement, de sorte que le schéma expérimental était un carré latin permettant une comparaison précise de ces 3 foins. Les 6 autres moutons non fistulisés ont reçu, selon le même schéma expérimental, les 3 pailles. Enfin, chacun des 6 fourrages a été distribué attx moutons fistulisés, les foins étant distribués successivement à 2 moutons, les pailles à 2 autres.

Tous les fourrages ont été distribués à volonté, à $8 \mathrm{~h}$ et à $16 \mathrm{~h}$ en 2 repas égaux. Les 3 pailles étaient complémentées en minéraux riches en $\mathrm{S}$ et $\mathrm{Zn}(20 \mathrm{~g} / \mathrm{jour})$ et la paille non traitée avec du tourteau de soja (100 g/jour). Une solution de vitamine $\left(\mathrm{AD}_{3} \mathrm{E}\right)$ a en outre été injectée aux moutons recevant les pailles au début de l'essai. Les fourrages ont été distribués durant 3 semaines aux animaux (15 jours d'adaptation, 6 jours de mesure), mais les moutons à la paille ont eu, en début d'essai, 7 jours d'adaptation de plus. 
Les différentes méthodes de mesures utilisées pour déterminer l'ingestibilité, la digestibilité, les caractéristiques de la digestion ruminale, l'activité cellulolytique dans le rumen, la vitesse de digestion des fourrages et la rétention azotée, sont les mêmes que celles décrites dans Dulphy et al. (1984) et Dulphy, Komar \& Zwaenepoel (1984). Il en est de même pour les analyses : cendres, matières azotées totales (MAT), cellulose brute (CB).

Pour préciser la forme de l'azote, nous avons, en outre, dosé la fraction soluble dans un tampon (méthode Durand, I.N.R.A., 1978), l'ammoniac libéré par extraction aqueuse à $+4^{\circ} \mathrm{C}$ pendant 18 heures (dosage par la méthode de ConwaY, 1957), ainsi que l'urée (méthode de Miches, non publiée). Cette méthode consiste à faire réagir l'urée à $85^{\circ} \mathrm{C}$ en présence de 2 solutions de réactifs (une solution avec $\mathrm{H}_{3} \mathrm{PO}_{4}$ et $\mathrm{FeCl}_{33}$; une autre avec de la diacétylemonoxime et de la thiosemicarbazine). Il se développe un complexe lu au colorimètre à $520 \mathrm{~nm}$. Le dosage est effectué par un analyseur automatique.

\section{Résultats}

\section{A. Composition chimique}

Les tencurs en MAT sont augmentées par les deux traitements (tabl. 1). Ceci est net pour le foin, mais l'amélioration a été plus faible pour la paille, en particulier pour celle traitée à l'urée, probablement parce que la paille a mal retenu la solution d'urée.

TABLEAL

Composition chimique des fourrages utilisés.

Chemical composition of different roughages.

\begin{tabular}{|c|c|c|c|c|}
\hline & \multirow{2}{*}{$\begin{array}{l}\text { Traitement } \\
\text { Treatment }\end{array}$} & \multicolumn{3}{|c|}{$\begin{array}{l}\text { Teneurs en } \mathrm{g} / 1000 \mathrm{~g} \text { de matière sèche } \\
\text { Content } \mathrm{g} / 1000 \mathrm{~g} \text { dry matter }\end{array}$} \\
\hline & & $\begin{array}{l}\text { Cendres } \\
\text { Ashes }\end{array}$ & $\begin{array}{c}\text { Matières azotées } \\
\text { totales } \\
\text { Crude protein }\end{array}$ & $\begin{array}{l}\text { Cellulose brute } \\
\text { Crude fibre }\end{array}$ \\
\hline \multirow{4}{*}{$\begin{array}{l}\text { Foin } \\
\text { Hay }\end{array}$} & $O \ldots \ldots \ldots \ldots$ & 65 & 117 & 364 \\
\hline & Urée $\ldots . . . \ldots$ & 60 & 228 & 353 \\
\hline & Urea & & & \\
\hline & $\mathrm{NH}_{3}, \ldots \ldots \ldots$ & 67 & 202 & 341 \\
\hline \multirow{3}{*}{$\begin{array}{l}\text { Paille } \\
\text { Straw }\end{array}$} & $O \ldots \ldots \ldots$ & 93 & $\begin{array}{l}28 \\
\text { (92 pour la } \\
\text { ration) }\end{array}$ & 459 \\
\hline & $\begin{array}{l}\text { Urée } \\
\text { Urea }\end{array}$ & 99 & 76 & 401 \\
\hline & $\mathbf{N H}: \ldots \ldots \ldots$ & 95 & 88 & 454 \\
\hline
\end{tabular}




\section{TABleau 2}

Fractionnement de l'azote contenu dans les fourrages étudiés. Levels of the different nitrogen fractions in the forages.

\begin{tabular}{|c|c|c|c|c|c|c|}
\hline & \multirow{3}{*}{$\begin{array}{l}\text { Traitement } \\
\text { Treatment }\end{array}$} & \multicolumn{5}{|c|}{$\begin{array}{l}\text { Teneurs en } \mathrm{g} \mathrm{N} / \mathrm{kg} \mathrm{MS} \\
\text { Levels in } \mathrm{g} \mathrm{N} / \mathrm{kg} \mathrm{DM}\end{array}$} \\
\hline & & \multirow{2}{*}{$\begin{array}{l}\text { Total } \\
\text { Total }\end{array}$} & \multicolumn{3}{|c|}{$\begin{array}{l}\text { Soluble } \\
\text { Soluble }\end{array}$} & \multirow{2}{*}{$\begin{array}{l}\text { Insoluble } \\
\text { Insoluble }\end{array}$} \\
\hline & & & $\begin{array}{l}\mathrm{NH}_{3} \\
\mathrm{NH}_{3} \\
\end{array}$ & $\begin{array}{l}\text { Urée } \\
\text { Urea }\end{array}$ & $\begin{array}{c}\text { Indéterminé } \\
\text { Indetermined }\end{array}$ & \\
\hline \multirow{3}{*}{$\begin{array}{l}\text { Foin } \\
\text { Hay }\end{array}$} & $0 \ldots \ldots$ & 18,72 & 0,46 & - & 3,70 & 14,56 \\
\hline & $\begin{array}{l}\text { Urée } \\
\text { Urea }\end{array}$ & 36,48 & 2,16 & 14,72 & 3,76 & 15,84 \\
\hline & $\mathrm{NH}_{;} \quad \ldots \ldots$ & 32,32 & 4,90 & - & 9,18 & 18,24 \\
\hline \multirow{3}{*}{$\begin{array}{l}\text { Paille } \\
\text { Straw }\end{array}$} & $0 \ldots \ldots$ & 4,48 & 0,43 & - & 1,33 & 2,72 \\
\hline & $\begin{array}{l}\text { Urée } \\
\text { Urea }\end{array}$ & 12,16 & 1,87 & 6,03 & 0,74 & 3,52 \\
\hline & $\mathrm{NH}_{3}$ & 14,08 & 4,69 & - & 4,43 & 4,96 \\
\hline
\end{tabular}

Les résultats du dosage des différentes fractions azotées sont donnés dans le tableau 2. Le traitement à l'urée augmente la quantité d'ammoniac présent dans le fourrage, mais peu par rapport au traitement à l'ammoniac lui-même. La transformation de l'urée en ammoniac a donc été peu efficace : 17 p. 100 pour le foin et 21 p. 100 pour la paille. Il faut noter aussi la forte augmentation de la fraction azote soluble indéterminé après traitement à l'ammoniac (complexes ammoniacaux ?).

Les teneurs en cendres et en cellulose brute n'ont, par contre, pas été modifiées notablement (tabl. 1).

\section{B. Ingestibilité (tabl. 3)}

Seul le traitement à l'ammoniac a amélioré l'ingestibilité : +26 p. 100 $(\mathrm{P}<0.05)$ dans le cas du foin, mais +9 p. 100 (non significatif) dans le cas de la paille. Le traitement à l'urée n'a pas augmenté l'ingestibilité du foin et a diminué celle de la paille $(-17$ p. $100, \mathrm{P}<0.05)$

\section{Digestibilité de la matière organique (DMO) et de la cellulose brute (tabl. 3)}

Le traitement à l'ammoniac a augmenté assez nettement les digestibilités de la matière organique $(+7,9$ points pour le foin et $+7,7$ pour la paille) et celles de la cellulose brute (respectivement $+11,2$ et $+8,9$ points). Par contre, le traitement à l'urée n'a eu qu'un très léger effet sur le foin $(+2,4$ points pour la DMO $)$ et pas d'effet sur la paille. 


\section{TABleaU 3}

Quantités ingérées et digestibilité des fourrages avant et après traitement ( 6 jours de mesure).

Intake and digestibility of forages before and after treatments

(6 days of measurements).

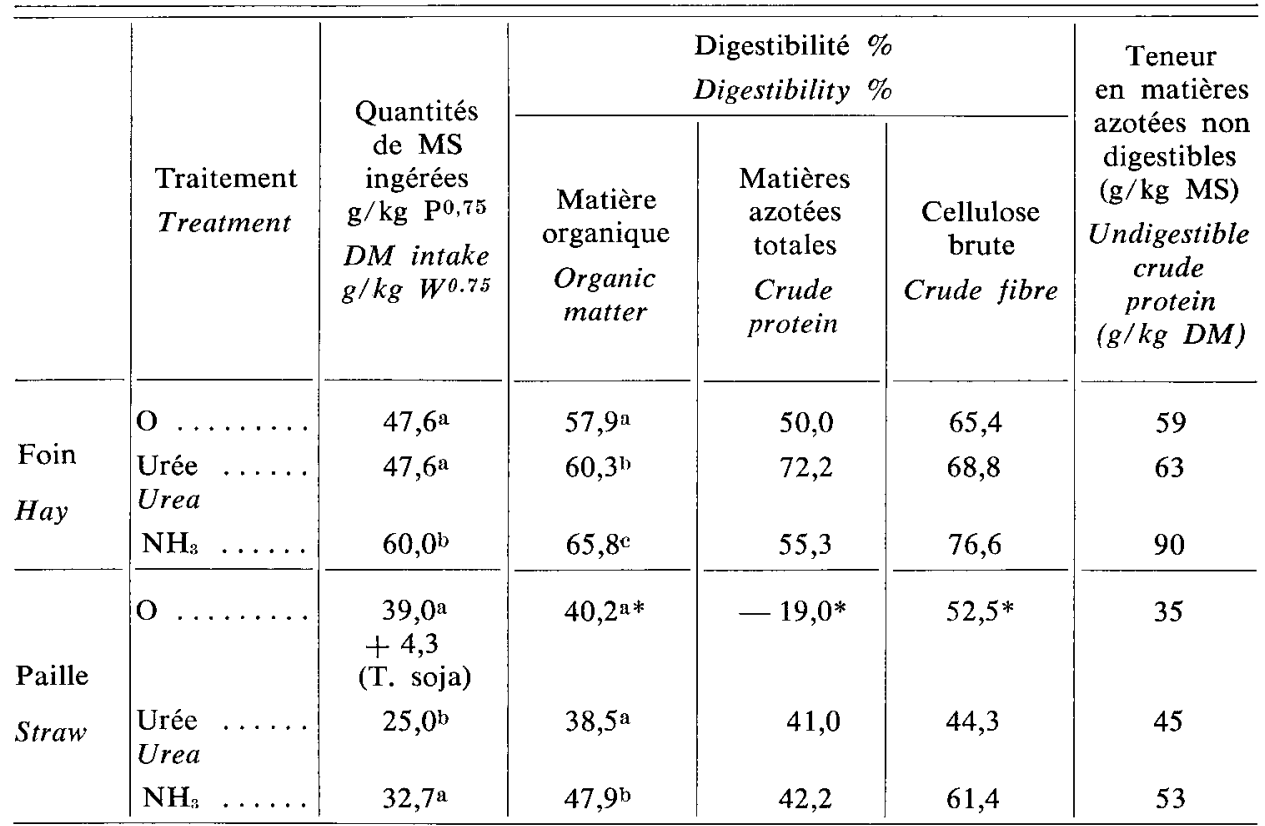

* Déduction faite du tourteau de soja - Except soyabean meal.

Syx $=2,5$ et 4,3 pour les quantités ingérées de foin et de paille.

2.5 and 4.3 for hay and straw intake.

Syx $=1,1$ et 3,9 pour les digestibilités de la MO de foin et de paille.

1.1 and 3.9 for $O M$ digestibility of hay and straw.

\section{Digestibilité des matières azotées (tabl. 3)}

La digestibilité apparente des MAT est améliorée par les 2 traitements, mais de façon moins importante que ne l'avait laissé espérer l'élévation de la teneur en MAT. En effet, les traitements se sont traduits par une augmentation des matières azotées non digestibles : +4 et $+10 \mathrm{~g} / \mathrm{kg}$ MS après traitement à l'urée, et +31 et $+18 \mathrm{~g} / \mathrm{kg}$ MS après traitement à l'ammoniac.

\section{E. Caractéristiques du jus de rumen}

1. $p H$ (tabl. 4 et fig. 1$)$

En moyenne, le traitement à l'urée n'a pas eu d'effet sur le $\mathrm{pH}$ du jus de rumen alors que celui à l'ammoniac l'a augmenté pour le foin $(+0,12)$ et abaissé pour la paille $(-0,18)$. 


\section{TABleau 4}

Caractéristiques moyennes du jus de rumen et digestibilité en sachets des fourrages avant et après traitement ( 2 jours de prélèvements).

Mean characteristics of rumen juice and in sacco digestibility of forages before and after treatment ( 2 days of measurements).

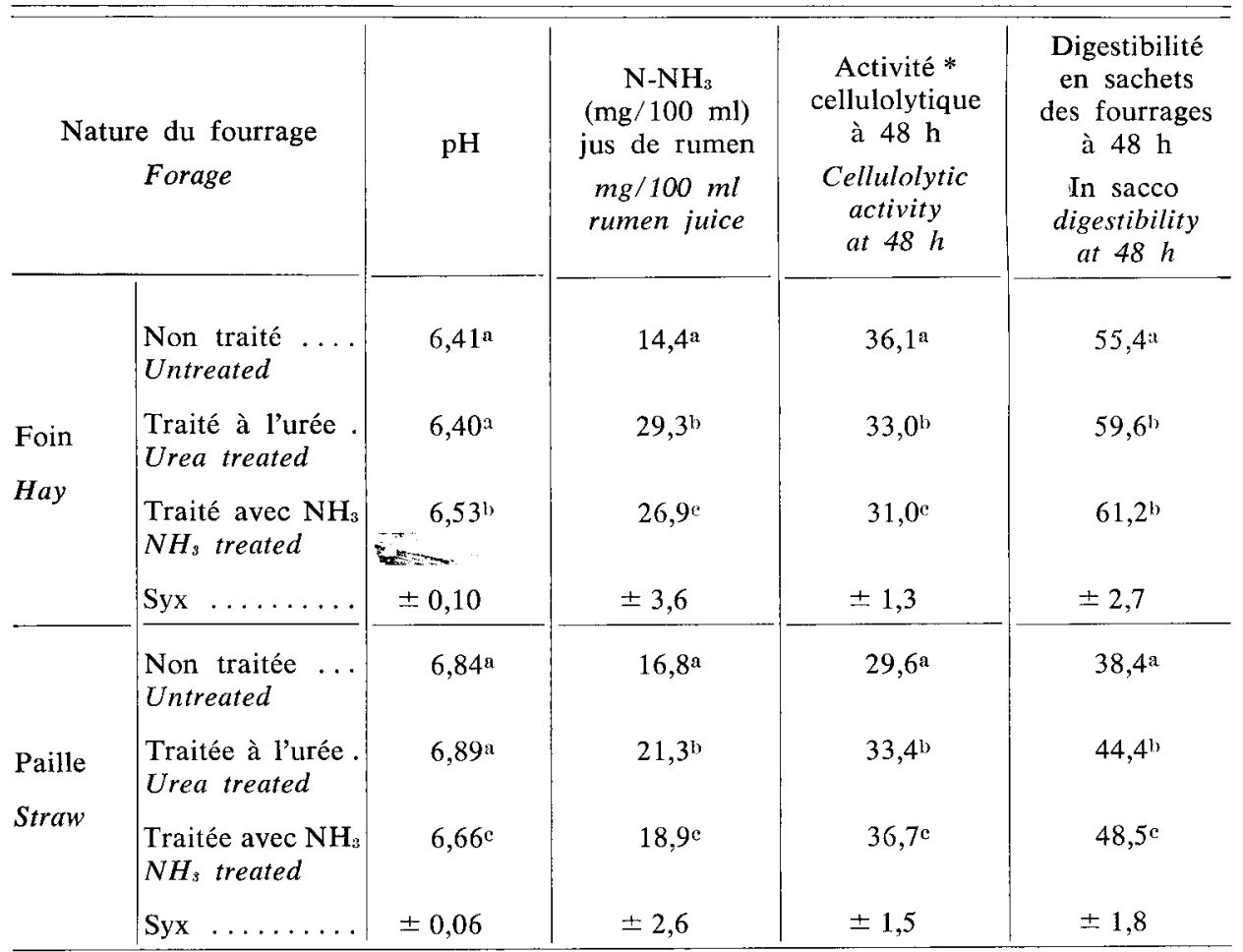

Activité cellulolytique $=$ digestibilité d'une paille témoin in sacco (p. 100). Cellulolytic activity $=$ in sacco digestibility of control straw (p. 100).

2. $\mathrm{NH}_{3}$ (tabl. 4 et fig. 2)

Les 2 traitements ont augmenté la teneur en $\mathrm{NH}_{3}$ du jus de rumen. Ceci a été plus net pour le foin que pour la paille, cette dernière ayant été complémentée avec du tourteau de soja lorsqu'elle n'était pas traitée. La valeur minimale atteinte a été de $9,7 \mathrm{mg} / 100 \mathrm{ml}$ pour la paille traitée à l'ammoniac. La teneur en $\mathrm{NH}_{3}$ dans le jus de rumen ne devrait pas avoir limité l'activité de la microflore. A l'inverse, il n'y a pas eu de teneurs excessives (teneur maximale de $36,9 \mathrm{mg} / 100 \mathrm{ml}$ pour le foin traité à l'ammoniac).

Notons également que la teneur en ammoniac dans le rumen a été plus élevée après le traitement à l'urée qu'après celui à l'ammoniac, surtout pour la paille, malgré une teneur en azote plus faible. 
(1)

(2)
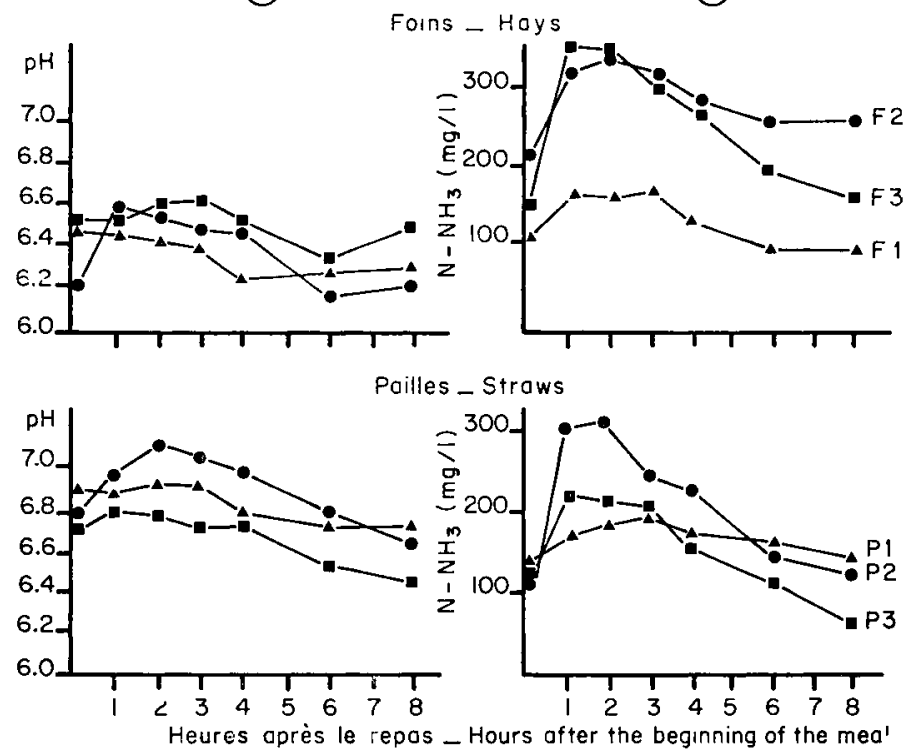

FIG. 1

Influence du traitement sur le pH du jus de rumen.

Effect of treatment on rumen $p H$.

FIG. 2

Influence du traitement sur la teneur en $\mathrm{N}-\mathrm{NH}_{z}$ du jus de rumen.

Effect of treatment on ammonia in rumen.

F1 : foin témoin - untreated hay.

F2 : foin + urée - hay + trea

$\mathrm{F} 3$ : foin $+\mathrm{NH}_{3}-h a y+\mathrm{NH}_{3}$

P1 : paille témoin - untreated straw

P2 : paille + uréc - straw + urea

P3 : paille $+\mathrm{NH}_{3}-s$ traw $+\mathrm{NH}_{\text {s }}$

3. Activité cellulolytique du jus de rumen (tabl. 4 et fig. 3)

Les foins et les pailles ont donné des résultats tout à fait contradictoires. En effet, les traitements ont abaissé l'activité cellulolytique pour les foins, mais l'ont augmenté pour les pailles. C'est le traitement à l'ammoniac qui a toujours eu l'effet le plus net. 


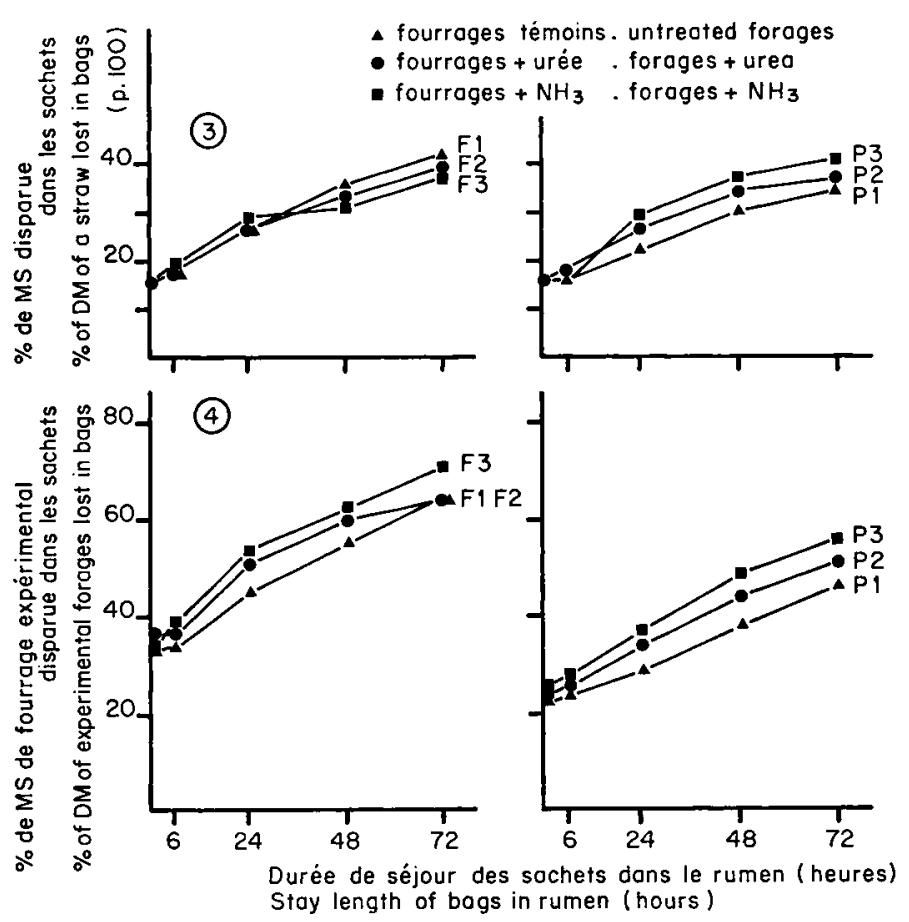

FIG. 3

Activité cellulolytique induite par les différents fourrages dans le rumen.

Cellulolytic activity induced by different types of forages in the rumen.

FIG. 4

Cinétique de digestion des fourrages dans le rumen des moutons recevant un foin de luzerne. Rate of digestion of forages in the rumen of sheep given lucerne hay.

F. Digestion in sacco des fourrages (tabl. 4 et fig. 4)

Globalement, l'importance de la digestion in sacco des fourrages a été améliorée de façon significative par les traitements, et plus par l'ammoniac que par l'urée.

\section{G. Rétention azotée (tabl. 5)}

L'azote ingéré a été fortement augmenté avec le foin après les traitements mais cela n'a pas été le cas pour la paille. L'azote fécal n'a pas été augmenté par le traitement à l'urée, mais il l'a été par celui à l'ammoniac. L'azote urinaire a été fortement augmenté avec le foin également par les 2 traitements, mais pas pour la paille. 


\section{Tableau 5}

Bilans azotés ( $\mathrm{g} /$ animal/jour).

Nitrogen balances ( $g /$ animal/day).

\begin{tabular}{|c|c|c|c|c|c|}
\hline \multicolumn{2}{|c|}{$\begin{array}{c}\text { Nature du fourrage } \\
\text { Forage }\end{array}$} & $\begin{array}{l}\mathrm{N} \text { ingéré } \\
\text { Ingested } \mathrm{N}\end{array}$ & $\begin{array}{c}\mathrm{N} \text { fécal } \\
\text { Faecal } N\end{array}$ & $\begin{array}{l}\mathrm{N} \text { urinaire } \\
\text { Urinary } N\end{array}$ & $\begin{array}{c}\mathrm{N} \text { retenu } \\
\text { Retained } N\end{array}$ \\
\hline \multirow{4}{*}{$\begin{array}{l}\text { Foin } \\
\text { Hay }\end{array}$} & $\begin{array}{l}\text { Non traité } \\
\text { Untreated }\end{array}$ & 18,0 & 9,0 & 9,2 & $-0,18^{n}$ \\
\hline & $\begin{array}{l}\text { Traité à l'urée } . . . \\
\text { Urea treated }\end{array}$ & 33,9 & 9,4 & 20,8 & $+3,73^{\prime \prime}$ \\
\hline & $\begin{array}{l}\text { Traité avec } \mathrm{NH}_{::} \\
\mathrm{NH}_{3} \text { treated }\end{array}$ & 38,7 & 17,3 & 21,5 & $-0,07 \AA$ \\
\hline & Syx $\ldots \ldots \ldots \ldots$ & & & & $\pm 2,09$ \\
\hline \multirow{4}{*}{$\begin{array}{l}\text { Paille } \\
\text { Straw }\end{array}$} & $\begin{array}{l}\text { Non traitée } \ldots \ldots \\
\text { Untreated }\end{array}$ & $10,9 *$ & 4,4 & 7,1 & $-0,66^{\mathrm{a}}$ \\
\hline & $\begin{array}{l}\text { Traitée à l'urée } \ldots \\
\text { Urea treated }\end{array}$ & 6,7 & 4,0 & 7,9 & $-5,15^{b}$ \\
\hline & $\begin{array}{l}\text { Traitée avec } \mathrm{NH}_{3} \ldots \\
\mathrm{NH}_{3} \text { treated }\end{array}$ & 10,9 & 6,3 & 7,3 & \\
\hline & Syx $\ldots \ldots \ldots \ldots \ldots$ & & & & $\pm 1,27$ \\
\hline
\end{tabular}

* Dont 7,7 pour le tourteau de soja.

$7.7 \mathrm{~g}$ for soyabean oil meal.

Finalement, cela s'est traduit par des résultats très différents pour le foin et la paille. En effet, le traitement à l'urée a amélioré le bilan azoté avec le foin $(+3,9 \mathrm{~g} /$ jour) mais pas avec la paille additionnée de tourteau de soja $(-4,5 \mathrm{~g} / \mathrm{jour})$. Celui à l'ammoniac ne l'a pas modifié avec le foin, mais l'a abaissé avec la paille $(-2 \mathrm{~g} /$ jour).

\section{Discussion - Conclusion}

Compte tenu de la composition chimique et de la valeur alimentaire des fourrages après traitement, il apparaît donc que le traitement du foin par l'ammoniac a été efficace dans la mesure où les résultats obtenus sont légèrement supérieurs à ceux rapportés par Dulphy et al. (1984). Par contre, le traitement de la paille à l'ammoniac n'a pas donné de très bons résultats (teneur en MAT inférieure à 10 p. 100 ; ingestibilité peu augmentée par rapport aux résultats obtenus antérieurement, mais augmentation correcte de la digestibilité - cf. DulPhy, Komar \& ZWAEnePOel, 1984).

Malgré une bonne fixation, l'urée a eu peu d'effet sur la valeur alimentaire du foin, ce qui est à relier à la faible uréolyse enregistrée. Pour la paille, le traitement 
à l'urée a donné des résultats franchement mauvais, dus à une mauvaise fixation de l'urée sur la paille qui n'était pas hachée et à une uréolyse faible.

Dans notre essai, le traitement à l'urée n'a pas eu d'effet sur la digestibilité de la matière organique, ni sur l'ingestibilité malgré une amélioration de la digestibilité des fourrages in sacco qui indique une légère action du traitement. Pour la digestibilité in vivo du foin, cela est peut-être à relier à la légère baisse d'activité cellulolytique dans le rumen, mais cette explication est probablement fausse, puisqu'on observe l'inverse avec la paille. L'absence d'effet sur l'ingestibilité est peut-être à relier au mauvais goût de l'urée. La technique du traitement à l'urée, lorsqu'elle est réussie, est pourtant prometteuse (MваtyA, 1983 ; Cloete \& Kritzinger, 1983), mais nous n'avons pas pu réunir les conditions nécessaires à un dégagement vigoureux d'ammoniac dans les tas de fourrage.

Les caractéristiques du jus de rumen des moutons recevant les différents fourrages n'ont pas présenté d'anomalies particulières : pas de $\mathrm{pH}$ très bas, teneur en ammoniac non limitante pour la microflore. A quelques détails près, les résultats correspondent à ceux que nous avons déjà obtenus (Dulphy et al., 1984 ; Dulphy, Komar \& ZWAEnePoel, 1984).

C'est plus au niveau de la valeur azotée des fourrages que nous discuterons nos résultats. Ils confirment tout d'abord la forte fixation d'azote sur le fourrage, azote qui se retrouve en partie dans les fécès, ce qui rejoint les observations des auteurs cités par Cloete \& Kritzinger (1984). Ceci a été particulièrement vrai pour le foin, dont la teneur en MAND était déjà élevée avant traitement, ce qui est le signe d'un échauffement léger lors de la conservation.

Pour le foin, les traitements ont entraîné une très forte perte d'azote dans l'urine, signe d'un large excédent d'azote ammoniacal dans le rumen. Cela se traduit, avec le traitement à l'ammoniac, par une rétention azotée inchangée malgré une très forte augmentation de l'azote ingéré $(+115 \mathrm{p}$. 100) et de la matière organique digestible ingérée $(+43$ p. 100) ce qui est tout à fait contradictoire avec les observations de Grenet \& Demarouilly (1982). Avec le traitement à l'urée, par contre, la rétention azotée a augmenté, probablement parce que la teneur en azote soluble du foin utilisé était trop faible au départ, cette hypothèse étant concordante avec celle d'un échauffement du foin utilisé, mais pas avec les teneurs en azote ammoniacal du jus de rumen qui étaient théoriquement correctes pour le développement de la microflore.

On peut penser également que l'urée qui se transforme dans le rumen est plus disponible que la fraction soluble indéterminée d'où un bilan positif, alors que pour le foin traité à l'ammoniac les fractions ammoniaquées sont moins disponibles.

Pour la paille, il est plus difficile de mesurer l'effet des traitements car la paille non traitée était distribuée avec du tourteau de soja, donc avec un aliment de valeur azotée élevée. Par ailleurs les moutons ne couvrant qu'environ la moilié de leurs besoins énergétiques d'entretien, la portée des mesures est très limitée. Néanmoins, pour des quantités d'azote ingérées identiques, la rétention azotée a été bien plus faible avec la paille traitéc à l'ammoniac qu'avec le régime paille non traitée associée à du tourteau de soja. Seule la fixation - de façon irréversible — d'une partie de l'ammoniac sur la paille semble en cause, mais dans l'étude précédente (DuLphy, Komar \& ZWaenepoel, 1984) il y avait aussi des pertes notables d'azote dans l'urine. 
Le fait que cela n'apparaisse pas dans cette nouvelle étude est lié peut-êtro à la faible teneur en azote de la ration. Avec le traitemnt à l'urée, la rétention azotée a été très faible, mais l'ingestion d'azote était faible et les pertes urinaires ont été relativement élevées, probablement par suite d'une faible synthèse bactérienne dans le rumen consécutive à une faible digestibilité.

Il apparaît donc que la valeur azotée des deux fourrages traités par l'ammoniac est mauvaise. Cela est dû tout d'abord au fait qu'une partie de l'azote ammoniacal reste fortement fixée sur les parois indigestibles (Yamara \& NumaKAwA, 1978 ; LAWlor \& O’Shea, 1979 ; Abidin \& Kempton, 1981 ; Gordon \& Chesson, 1983). Cela se voit au niveau des augmentations en azote insoluble de nos fourrages après traitement qui expliquent en grande partie celles en azote dans les fécès. L'accroissement de la teneur en azote des fécès pourrait être dû également au fait qu'une partie des protéines présentes dans le fourrage est insolubilisée lors du traitement par la chaleur de réaction de l'ammoniac sur les parois végétales. La mauvaise valeur azotée de nos fourrages traités est due aussi à une perte élevée d'azote urinaire provenant d'un excès d'azote ammoniacal dans le rumen par rapport aux besoins de la microflore. Là se pose le problème de l'efficacité de la synthèse bactérienne. En effet, le traitement à l'ammoniac induit une augmentation, parfois nette, de la teneur en matière organique digestible qui devrait s'accompagner d'une synthèse bactérienne accrue (I.N.R.A., 1978) et donc d'une rétention azotée plus élevée (Grenet \& Demarouilly, 1982). Or ce n'est pas le cas. La faiblesse de l'activité cellulolytique trouvée par ailleurs par Dulphy et al. (1984) et pour le foin de cet essai pourrait l'expliquer. Reste à savoir pourquoi avec la paille de cet essai l'activité cellulolytique dans le rumen a augmenté après le traitement à l'ammoniac?

En conclusion, après avoir confirmé nos observations, il sera nécessaire de mettre au point un système de correction pour le calcul de la valeur azotée des fourrages traités à l'ammoniac. Il sera très important également de rechercher les compléments (fourrages ou concentrés) les plus adaptés pour tirer cependant partie de la quantité d'azote élevée contenue dans ces fourrages.

\section{Summary}

\section{Nitrogen value of poor forages treated with urea or aimmonia}

Late maturing natural-meadow hay and wheat straw were treated (under sheet) with ammonia (3 p. 100) or urea (5 p. 100). Untreated and treated forages were offered to normal or rumen-fistulated shecp. Animals receiving untreated wheat straw were also given $100 \mathrm{~g}$ soyabean oil-meal per day.

Ammoniation resulted in a marked rise in forage crude protcin content $(+85$ and $+60 \mathrm{~g} / \mathrm{kg} \mathrm{DM})$, feed intake $(+26$ and +9 p. 100) and organic matter digestibility $(+7.9$ and +7.7 points).

Urea treatment was not efficient. The forage crude protein content increased $(+111$ and $+48 \mathrm{~g} / \mathrm{kg} \mathrm{DM}$ ), but the straw intake decreased by $17 \mathrm{p} .100$ and the organic matter digestibility remained unchanged. This was due to a poor transformation of urea into ammonia (on an average only 19 p. 100 for the two forages).

Treatments, especially with ammonia, reduced the cellulolytic activity in the rumen in the case of hay, but the opposite was observed with straw (Table 4, fig. 3) ; the digestion rate of straw increased, but not that of hay (fig. 2). Treatments also led to a higher ammonia concentration in the rumen (Table 4, fig. 1). 
Ammonia treatment led to a lower nitrogen retention in the animals.

Thus, it seems that the nitrogen nutritive value of ammonia treated forages is rather low despite a large nitrogen supply. However, the cellulolytic activity is perhaps not reduced by the treatment in all cases.

Urea treatments are developing and give positive results; they should be adapted to our forage and treatment conditions.

Key words : Hay, straw, ammonia treatment, feeding value, nitrogen retention.

Reçu en janvier 1985.

Accepté en mai 1985.

\section{Références bibliographiques}

Abidin Z., Kempton T.J., 1981. Effects of treatment of barley straw with anhydrous ammonia and supplementation with heat-treated protein meals on feed intake and liveweight performance of growing lambs. Anim. Feed Sci. Technol., 6, 145-155.

Cloete S.W.P., Kritzinger M.N., 1984. Urea ammoniation compared to urea supplementation as a method of improving the nutritive value of wheat straw for sheep. S. Afr. J. Anim. Sci., 14, 59-63.

Cloete S.W.P., De Vili.iers T.T., Kritzinger N.M., 1983. The effect of ammoniation by urea on the nutritive value of wheat straw for sheep. S. Afric. J. Anim. Sci., 13, 143-146.

Conway E.J., 1957. In Microdiffusion analysis and volumetric error. Crosby, Lockwood, London.

Dulphy J.P., Komar A., Zwaenepoel P., 1984. Effets comparés des traitements à l'ammoniac et à la soude sur la valeur alimentaire de fourrages pauvres. Ann. Zootech., 33, 321-342.

Dulphy J.P., Zwaenepoel P., Komar A., Aboulfaraj S., 1984. Valeur alimentaire des foins traités par l'ammoniac. Ann. Zootech., 33, 187-200.

Grenet E., Demarquilly C., 1982. Utilization of nitrogen from fresh forage, silage and hay by growing sheep. Proc. 9th General Meeting European Grassland Federation. Reading (G.-B.), 5-9 Sept., 241-245.

Gordon A.H., Chesson A., 1983. The effect of prolonged storage on the digestibility and nitrogen content of ammonia-treated barley straw. Anim. Feed. Sci. Technol., 8, 147153.

Hadipanayiotou M., 1982. The effect of ammoniation using urea on the intake and nutritive value of chopped barley straw. Grass Forage Sci., 37, 89-93.

I.N.R.A., 1978. Alimentation des ruminants. Ed. I.N.R.A. Publications, route de Saint-Cyr, 78000 Versailles, France. 594 pages.

JaCKSON M.G., 1977. Review article : the alcali treatments of straw. Anim. Feed Sci. Technol., 2, 105-130.

LAwlor M.J., O'SHEA J., 1979. The effect of ammoniation on the intake and nutritive value of straw. Anim. Feed Sci. Technol., 4, 169-175.

MbatYa P.B.A., 1983. Methods of improving the utilization of cereal straw by ruminants. III -A note on the effect of ensiling straw treated with urea. Anim. Feed Sci. Technol., 9, $181-183$.

Sundstel F., Coxworth E., Mowat D.N., 1978. Improving the nutritive value of straw and other low quality roughages by treatment with ammonia. World Anim. Review, 26, $13-21$.

YamaRa N., Numakawa T., 1978. Improvement of preservation and quality of semi-dried hay by anhydrous ammonia treatment. Jap. J. Zootech. Sci., 49, 648-652. 